\title{
EVALUATION OF CLINICALLY IMPORTANT GRAM POSITIVE ISOLATES AND THEIR SUSCEPTIBILITY PATTERN IN TERTIARY CARE HOSPITALS: 3-YEAR PROSPECTIVE STUDY
}

\author{
Ram Murugan Navaneethakrishnan'1, Sugumari Chandrasegaran ${ }^{2}$ \\ ${ }^{1}$ Senior Assistant Professor, Department of Microbiology, Madurai Medical College, Madurai. \\ ${ }^{2}$ Senior Assistant Professor, Department of Microbiology, Madurai Medical College, Madurai.
}

\section{ABSTRACT}

AIM

1. To analyse clinically important Gram-Positive isolates (Staphylococcus aureus, Enterococcus Species and Streptococcal Species) and their susceptibility pattern in tertiary care Hospitals. 2. To study the epidemiology of the isolates and sensitivity pattern of Gram Positive isolates in South Tamilnadu.

\section{METHODS}

Samples collected from different wards were subjected to culture, isolation and identification of Gram Positive isolates and Antibacterial Susceptibility testing by Kirby Bauer method. This study was carried out in the Institute of Microbiology, Madurai Medical College, Madurai.

\section{RESULTS}

6900 non-repetitive isolates from 29,275 patients were collected. They were from urine, sputum, blood, pus and respiratory samples. Total number of Gram Positive isolates processed was 1022. Staphylococcus aureus was the most common organism isolated in our study followed by Enterococcus Species and Streptococcal Species. Staphylococcus aureus isolates showed high sensitivity to Glycopeptides, Clindamycin, Doxycycline, Cefazolin, Erythromycin and Amoxicillin-Clavulanic acid. This isolates showed low sensitivity to Penicillin, Trimethoprim/Sulphonamides and Ciprofloxacin. Enterococcus isolates showed high sensitivity to Glycopeptides. These isolates showed low sensitivity to Penicillin, Amoxicillin-Clavulanic acid, Ampicillin, Nitrofurantoin, Levofloxacillin, High Level Gentamicin and High Level Streptomycin. Streptococcus isolates showed high sensitivity to Penicillin, Clindamycin, Doxycycline, Erythromycin, Ofloxacillin and Ceftriaxone. Methicillin Resistant Staphylococcus aureus showed resistance from 5\% to $4.20 \%$. Vancomycin Resistant Enterococci showed resistance from $0.50 \%$ to $0 \%$.

\section{CONCLUSION}

In this 3-year study, Staphylococcus aureus was the most common organism isolated from clinical specimens. Staphylococcus aureus isolates showed high sensitivity to Glycopeptides Clindamycin, Doxycycline, Cefazolin, Erythromycin and AmoxicillinClavulanic acid. Enterococcus isolates showed high sensitivity to Glycopeptides. Methicillin Resistant Staphylococcus aureus and Vancomycin-Resistant Enterococci exhibit lesser resistance rates in the 3-year study period. This study will help both clinicians and infection controlling practitioners to treat the patients. This single center study can give important information regarding emerging resistance patterns.

\section{KEYWORDS}

Staphylococcus Aureus, Enterococcus, Streptococcus, Methicillin Resistant Staphylococcus Aureus, Vancomycin Resistant Enterococci.

HOW TO CITE THIS ARTICLE: Navaneethakrishnan RM, Chandrasegaran S. Evaluation of clinically important gram positive isolates and their susceptibility pattern in tertiary care hospitals: 3-year prospective study. J. Evolution Med. Dent. Sci. 2016;5(57):38853889, DOI: $10.14260 /$ jemds/2016/890

\section{INTRODUCTION}

Gram-positive cocci are becoming increasingly resistant to traditionally used antimicrobial agents. Staphylococcus aureus and Enterococci are the most commonly isolated bacteria causing (Nosocomial) infections. Among those giving therapeutic problems are methicillin-resistant staphylococci and Vancomycin-resistant enterococci. ${ }^{1}$ Staphylococcus aureus is still one of the five most common causes of hospitalacquired infections. S. aureus is one of the most common causes of bacteraemia and acute infective endocarditis.

Financial or Other, Competing Interest: None.

Submission 08-06-2016, Peer Review 03-07-2016,

Acceptance 08-07-2016, Published 15-07-2016.

Corresponding Author:

Dr. Ram Murugan Navaneethakrishnan,

\#14/2, Siva Sakthi Nagar,

K. Pudur, Madurai-625007.

E-mail: rammddo@yahoo.co.in,doctorsugumari@yahoo.com

DOI: $10.14260 /$ jemds $/ 2016 / 890$
Additionally, it can cause various skin and soft tissue infections. ${ }^{2}$ MRSA is any strain of Staphylococcus aureus that has developed through the process of natural selection, multiresistance to beta-lactam antibiotics, which include the penicillins (Methicillin, Dicloxacillin, Nafcillin, Oxacillin, etc.) and the Cephalosporins. The most recent HealthcareAssociated Infections Progress Report of CDC published in 2016 reported a 13 percent decrease in MRSA bacteraemia between 2011 and 2014 in national acute care hospitals' report survey.

Methicillin Resistant Staphylococcus aureus (MRSA) is now endemic in India. The incidence of MRSA varies from 25 percent in western part of India to 50 percent in South India. 3,4 Very few cases of Vancomycin-intermediate S. aureus/Vancomycin-resistant S. aureus (VISA/VRSA) in India are reported. ${ }^{5}$ Enterococcus faecalis and Enterococcus faecium are the most prevalent species cultured from humans, accounting for more than $90 \%$ of clinical isolates. 
National Nosocomial Infections Surveillance (NNIS) data reveal the pooled mean for Vancomycin-Resistant Enterococcus (VRE) species from all ICUs, non-ICU inpatient areas and outpatient areas were $13.9 \%, 12 \%$ and $4.6 \%$ respectively from 1998 through June 2004. Pneumococcal bacteria are resistant to one or more antibiotics in $30 \%$ of cases. 6 No resistance of Streptococcus pyogenes to Penicillin has been reported to date, although since 1985 many reports of Penicillin tolerance have been made.

\section{MATERIAL AND METHODS \\ Type of Study \\ Cross-sectional, observational, prospective study.}

\section{Settings}

The study was conducted in a tertiary care teaching hospital.

\section{Sample Size}

6900 non-repetitive isolates from 29,275 patients. They were collected from urine, sputum, blood, pus and respiratory samples. Total number of Gram Positive isolates processed was 1022.

\section{Study Period}

From 2013-2015.

\section{Inclusion Criteria}

Newly admitted patients without any antibiotic treatment.

\section{Exclusion Criteria}

Patients already with antibiotic treatments.

\section{METHODOLOGY}

We analysed microbiologically important Gram-Positive isolates (Staphylococcus aureus, Streptococcus species, Enterococcus) and their susceptibility patterns. The study began in 2013 and continued up to 2015. Clinical data were prospectively collected by local infection control team using a standardized case-report form. The data that were routinely collected included the patient's age, sex, locations namely ICU, non-ICU ward and outpatient patients. The antimicrobial susceptibility patterns of 6900 non-repetitive isolates from 29,275 was studied. Total number of Gram positive coccus isolates processed was 1022. Subsequent cultures of the same organism from the same patient were not included. Samples such as Blood, Urine, Pus, Respiratory samples were collected from patients attending as outpatients and also from different clinical departments, wards and intensive care units by observing standard specimen collection procedure and aseptic conditions. The samples were transported to the lab and streaked on MacConkey Agar and Blood Agar medium. After the incubation period at $37^{\circ} \mathrm{C}$, the culture plates were examined and bacterial isolates were observed by Gram staining and motility test. On Gram staining if Gram positive cocci appeared as pairs, Catalase test was performed. If it was Positive, Slide and Tube Coagulase tests were performed. It was identified as Staphylococcus species. If Gram positive cocci appeared as chains or as diplococci, Catalase tests were performed. If it was negative, it was identified as Streptococcus Species. The identification of bacteria was performed by standard protocol (On observing Blood Agar Plates, Opaque Colonies and type of haemolysis were observed).
Alpha haemolytic colonies were processed with optochin sensitivity and bile solubility test. If optochin test and bile solubility was positive, the organisms were identified as Pneumococci. If those tests were negative, they were identified as Viridans Streptococci. Beta haemolytic colonies were processed with modified oxidase test, Coagulase test, Bacitracin Sensitivity, Novobiocin Sensitivity and Camp test. If Modified Oxidase test was positive, it was identified as Micrococci. If Tube and Slide Coagulase tests were positive, it was identified as Staphylococcus aureus. If Novobiocin sensitivity test was positive, it was identified as Staphylococcus saprophyticus. If Bacitracin Sensitivity test was positive, it was identified as Streptococcus pyogenes.

If Camp test was positive, it was identified as group B Streptococci. Non-haemolytic (Gamma) colonies were tested with Bile Esculin test, Heat tolerance test and growth in 6.5\% $\mathrm{NaCl}$ and identified as Enterococcus species. If Cefoxitin sensitivity test was positive, it was identified as Methicillin Sensitive Staphylococcus aureus (MSSA). Cefoxitin is an even better inducer of the mecA gene, and tests using cefoxitin give more reproducible and accurate results than tests with oxacillin. Cefoxitin disc is added with other routinely used antibiotics for MRSA confirmation.

If it was negative, it was identified as Methicillin Resistant Staphylococcus aureus (MRSA). Vancomycin Sensitivity test was done with Enterococcus species by E Test Strip. If the test was negative, it was identified as Vancomycin Resistant Enterococci (VRE). Enterococcal organisms for which the vancomycin zone diameters are $>=15 \mathrm{~mm}$ are considered susceptible, although several studies showed that this breakpoint is unreliable for detecting VISA strains. So Disc Diffusion test method with Vancomycin for enterococci is not done.

Susceptibility testing was done with the disc diffusion method (Modified Kirby-Bauer method) using Mueller-Hinton agar. The results were interpreted as per CLSI (Clinical and Laboratory Standards Institute) guidelines corresponding to that period. All antibiotic discs were obtained from OXOID (Oxoid Ltd., Altrincham, Cheshire, United Kingdom) and BD BBL (Becton, Dickinson and Company Ltd., Franklin Lakes, New Jersey, USA). MRSA, VRSA and VRE were interpreted based on Kirby-Bauer disc susceptibility testing as per CLSI guidelines.7,8 MIC (E Test) for Gram positive Cocci with Linezolid and Daptomycin (Second Line Drugs) were only being done.

We obtained Institutional Ethics Committee clearance for the study.

\section{RESULTS}

\begin{tabular}{|c|c|c|c|c|}
\hline Year & $\mathbf{2 0 1 3}$ & $\mathbf{2 0 1 4}$ & $\mathbf{2 0 1 5}$ & Total \\
\hline Total no. of Samples & 8955 & 9628 & 10692 & 29275 \\
\hline Total nos. Positive & 2978 & 2053 & 1869 & 6900 \\
\hline Percentage & 33 & 21.30 & 17.40 & 24 \\
\hline \multicolumn{6}{|c|}{ Table 1: Isolation of Microorganisms from } \\
Samples (2013-2015) \\
\hline
\end{tabular}

Table 1 shows, out of 29275 samples 6900 isolates were made. The isolates in 2013, 2014, 2015 found 2978 (33\%), 2053 (21.30\%), 1869(17.40\%) respectively. 


\begin{tabular}{|c|c|c|c|c|c|c|c|c|c|c|c|c|}
\hline \multicolumn{13}{|c|}{ Predominant Isolates - Sample-Wise $\mathrm{n}=1022$} \\
\hline \multirow[t]{2}{*}{ Isolates } & \multicolumn{3}{|c|}{ Urine } & \multicolumn{3}{|c|}{ Blood } & \multicolumn{3}{|c|}{ Respiratory } & \multicolumn{3}{|c|}{ Pus } \\
\hline & $\stackrel{m}{\stackrel{\sim}{2}}$ & 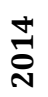 & $\stackrel{\text { no }}{\underset{\sim}{2}}$ & $\stackrel{n}{\stackrel{\sim}{\sim}}$ & $\underset{⿱ 亠}{\stackrel{J}{j}}$ & 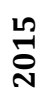 & $\stackrel{n}{\stackrel{n}{*}}$ & $\underset{\text { ¿ }}{\stackrel{+}{j}}$ & $\stackrel{\text { L }}{\underset{7}{2}}$ & $\stackrel{m}{\stackrel{n}{0}}$ & $\underset{\text { J }}{\stackrel{J}{d}}$ & 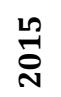 \\
\hline Staphylococcus aureus & 34 & 6 & 8 & 16 & 9 & 11 & 23 & 9 & 5 & 166 & 102 & 136 \\
\hline Enterococcus & 156 & 77 & 50 & 3 & 10 & 11 & 3 & 0 & 13 & 11 & 62 & 36 \\
\hline Streptococcus & 0 & 0 & 1 & 0 & 7 & 3 & 16 & 12 & 3 & 4 & 7 & 12 \\
\hline
\end{tabular}

Table 2 shows out of 1022 samples, Staphylococcus aureus grown in 525, Enterococcus Species grown in 432 and Streptococcal Species grown in 65 samples.

\begin{tabular}{|c|c|c|c|}
\hline Antibiotics & 2013 & 2014 & 2015 \\
\hline Glycopeptides & 100 & 100 & 100 \\
\hline Penicillin & 19.7 & 9 & 14 \\
\hline Erythromycin & 51.3 & 66 & 90 \\
\hline Cefoxitin & 73 & 89 & 96 \\
\hline Co-trimoxazole & 37 & 21 & 14.10 \\
\hline Clindamycin & 74 & 84 & 98.70 \\
\hline Doxycycline & 93.4 & 99 & 98.70 \\
\hline Cefazolin & 77.6 & 98 & 96.90 \\
\hline Ciprofloxacin & 71 & 43 & 36.90 \\
\hline Amoxi-clavulanic acid & 0 & 63 & 71.40 \\
\hline \multicolumn{4}{|c|}{$\begin{array}{c}\text { Table } 3 \text { (Chart 1): Sensitivity Pattern of Gram Positive } \\
\text { Antibiotics for Staphylococcus aureus in } \\
\text { Percentage } n=1022\end{array}$} \\
\hline
\end{tabular}

Table 3 (Chart 1) shows the Sensitivity pattern of Gram Positive Antibiotics for Staphylococcus aureus in percentage $\mathrm{n}=1022$.

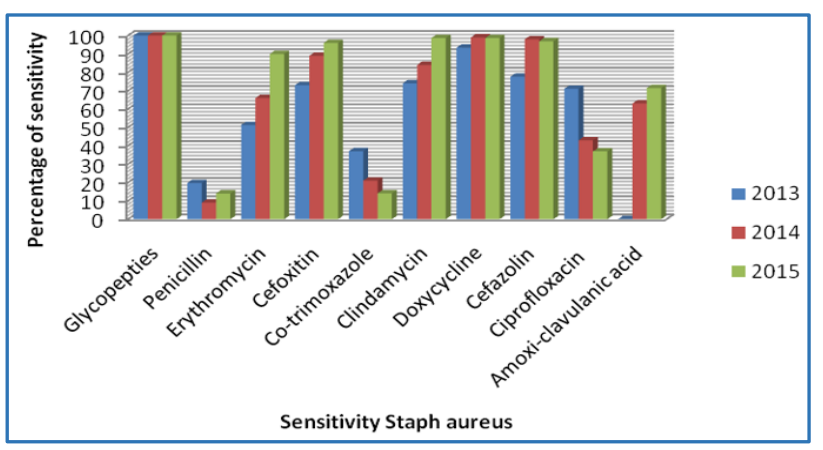

Table 3 (Chart 1) shows that Staphylococcus aureus isolates had high sensitivity to Glycopeptides (100\%), Clindamycin from $74 \%$ to $98.7 \%$, Doxycycline $93.4 \%$ to $98.7 \%$, Cefazolin $77.6 \%$ to 96.90 , Erythromycin $51.3 \%$ to $90 \%$, and Amoxicillin-Clavulanic acid $63 \%$ to $71.40 \%$ ). The isolates showed low sensitivity to Penicillin from $(19.7 \%$ to $14 \%)$ Trimethoprim/Sulphonamides $\quad(37 \%$ to $14.1 \%)$ and Ciprofloxacin (71\% to $36.90 \%$ ).

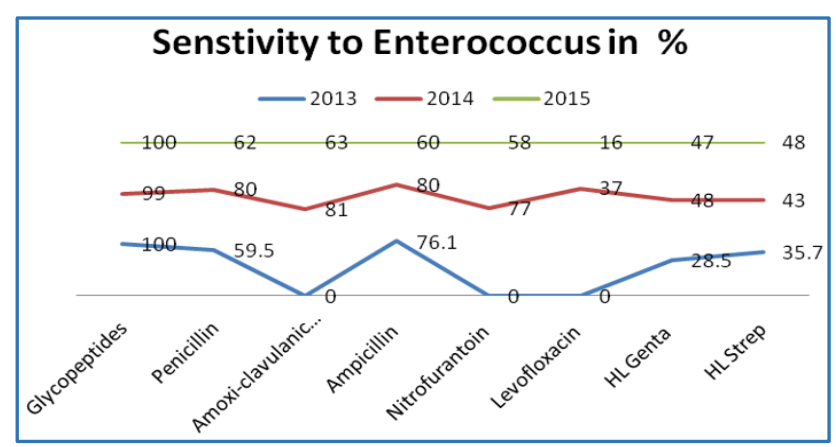

\begin{tabular}{|c|c|c|c|}
\hline \multicolumn{4}{|c|}{$\begin{array}{c}\text { Sensitivity Pattern of Gram Positive Antibiotics for } \\
\text { Enterococcus in Percentage n =1022 }\end{array}$} \\
\hline Antibiotics & 2013 & 2014 & 2015 \\
\hline Glycopeptides & 100 & 99 & 100 \\
\hline Penicillin & 59.5 & 80 & 62 \\
\hline Amoxicillin-clavulanic acid & 0 & 81 & 63 \\
\hline Ampicillin & 76.1 & 80 & 60 \\
\hline Nitrofurantoin & 0 & 77 & 58 \\
\hline Levofloxacin & 0 & 37 & 16 \\
\hline HL Genta & 28.5 & 48 & 47 \\
\hline HL Strep & 35.7 & 43 & 48 \\
\hline
\end{tabular}

Table 4 (Chart 2): Sensitivity Pattern of Gram Positive Antibiotics for Enterococcus Species

Table 4 (Chart 2) shows that, Enterococcus isolates were high sensitivity to Glycopeptides (100\%),). The isolates showed low sensitivity to Penicillin from (59.5\% to 62\%), Amoxicillin-Clavulanic Acid (81\% to 63\%), Ampicillin (76.1\% to $60 \%$ ), Nitrofurantoin ( $77 \%$ to $58 \%$ ), Levofloxacillin (37\% to 16\%) High Level Gentamicin (28.5 to 47\%) and High Level Streptomycin (35.7 to $48 \%$ ).

\begin{tabular}{|c|c|c|c|}
\hline \multicolumn{4}{|c|}{$\begin{array}{l}\text { Sensitivity Pattern of Gram Positive Antibiotics for } \\
\text { Streptococcus in Percentage. } n=1022\end{array}$} \\
\hline Antibiotics & 2013 & 2014 & 2015 \\
\hline Penicillin & 96 & 100 & 100 \\
\hline Erythromycin & 89 & 100 & 100 \\
\hline Clindamycin & 98 & 100 & 100 \\
\hline Doxycycline & 97 & 90 & 91 \\
\hline Ofloxacin & 63 & 88 & 90.30 \\
\hline Ceftriaxone & 65 & 90 & 97 \\
\hline \multicolumn{4}{|c|}{$\begin{array}{c}\text { Table } 5 \text { (Chart 3): Sensitivity Pattern of Gram Positive } \\
\text { Antibiotics for Streptococcus Species }\end{array}$} \\
\hline
\end{tabular}

Table 5 (Chart 3) shows that Streptococcus isolates were high sensitivity to Penicillin (96\% to $100 \%)$, Clindamycin from (98\% to $100 \%)$, Doxycycline (97\% to $91 \%$ ), Erythromycin ( $89 \%$ to $100 \%$ ), Ofloxacillin (63\% to $90.30 \%$ ) and Ceftriaxone (65\% to $97 \%)$.

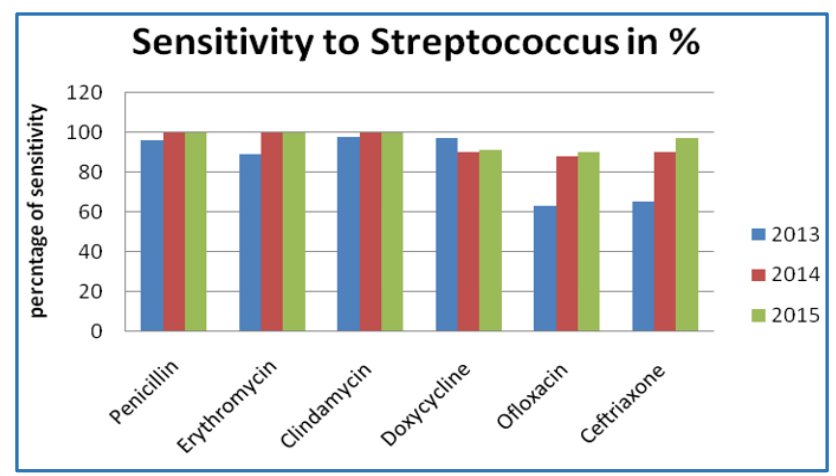




\begin{tabular}{|c|c|c|c|}
\hline \multicolumn{4}{|c|}{ Comparison of MRSA and VRE in Percentage } \\
\hline & $\begin{array}{c}\text { Year } \\
\mathbf{2 0 1 3}\end{array}$ & $\begin{array}{c}\text { Year } \\
\mathbf{2 0 1 4}\end{array}$ & $\begin{array}{c}\text { Year } \\
\mathbf{2 0 1 5}\end{array}$ \\
\hline $\begin{array}{c}\text { Methicillin Resistant } \\
\text { Staphylococcus aureus }\end{array}$ & 5.00 & 12.20 & 4.20 \\
\hline $\begin{array}{c}\text { Vancomycin Resistant } \\
\text { Enterococcus }\end{array}$ & 0.50 & 1.20 & 0.00 \\
\hline \multicolumn{4}{|c|}{ Table 6 (Chart 4): MRSA and VRE in Percentage } \\
\hline
\end{tabular}

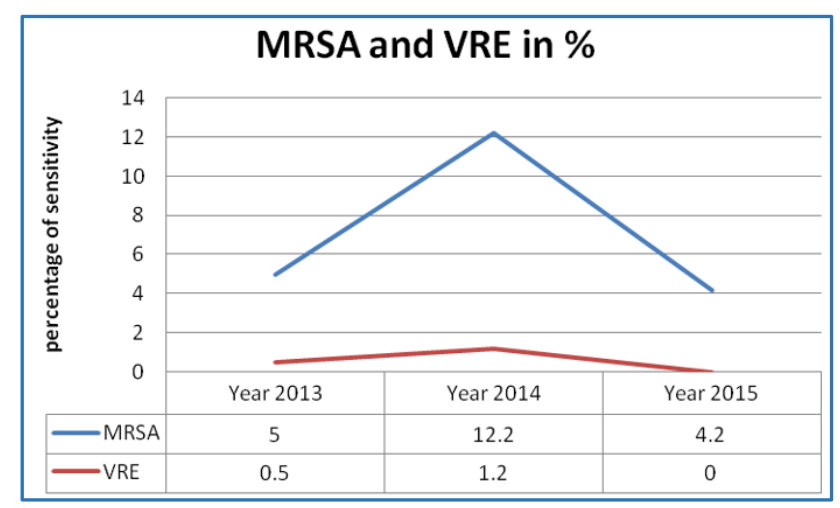

Table 6 (Chart 4) shows that Methicillin Resistant Staphylococcus aureus were from $5 \%$ to $4.20 \%$. Vancomycin Resistant Enterococci were from $0.50 \%$ to $0 \%$. Statistical analysis or $\mathrm{P}$ value has no relevance in this study.

\section{DISCUSSION}

In our study Staphylococcus aureus isolates were highly sensitive to Glycopeptides, Clindamycin, Doxycycline, Cefazolin, Erythromycin and Amoxicillin-Clavulanic acid. These isolates showed low sensitivity to Penicillin, Trimethoprim/Sulphonamides and Ciprofloxacin. An Indian study demonstrated that susceptibility to Ciprofloxacin was low in both MSSA (53\%) and MRSA (21\%). ${ }^{9}$ MSSA isolates showed a higher susceptibility to Gentamicin, Co-trimoxazole, Erythromycin and Clindamycin as compared to MRSA isolates. No isolate was found to be resistant to Vancomycin or Linezolid. This study correlates highly with our study. Nutanbala N Goswami et al had demonstrated the Staphylococcus aureus isolates were high sensitivity to Rifampicin, Clindamycin, Levofloxacin and Vancomycin, and were resistant to Penicillin (29.82\%) and Gentamicin (29.82\%).10 This study correlates highly with our study.

In our study, Enterococcus isolates showed 100\% sensitivity to Glycopeptides. These isolates showed low sensitivity to Penicillin, Amoxicillin-Clavulanic acid, Ampicillin, Nitrofurantoin, Levofloxacillin, HL Gentamicin and HL Streptomycin. Purva Mathur et al noted that Vancomycin resistance was found in $1 \%$ of isolates. ${ }^{11}$ Enterococcus showed resistance to Aminoglycosides 26\%, Ampicillin 66\%, Ciprofloxacin 88\% and Erythromycin 85\%. This study correlates highly with our study. Sreeja et al noted that the sensitivity pattern of these isolates showed an increased resistance to Penicillin, Ampicillin and Ciprofloxacin. ${ }^{12}$ A High Level of Gentamicin Resistance (HLGR) was present in 60 (47\%) isolates of Enterococcus. This study correlates highly with our study.

In our Study, Streptococcus isolates were high sensitivity to Penicillin, Clindamycin, Doxycycline, Erythromycin, Ofloxacillin and Ceftriaxone. R. Ratheesh et al showed that
Streptococcus pneumonia showed $100 \%$ sensitivity to Penicillin and Erythromycin, 78.3\% to Co-trimoxazole, 77.2\% to Ceftriaxone, $23.8 \%$ to Clindamycin and $24.3 \%$ to Gentamicin. ${ }^{13}$ This study correlates well with our study.

In this study shown that sensitivity pattern of Streptococcus to various antibiotics in 2014 and 2015 is more than in 2013 because of improvement in infection control practices like hand washing, disinfection and antibiotic policy. This is the main cause for decreasing resistance in 2014 and 2015.

In our study, Methicillin Resistant Staphylococcus aureus were from 5\% to $4.20 \%$. Vancomycin Resistant Enterococci were from $0.50 \%$ to $0 \%$. AP Mehta et al showed a marked decrease in MRSA isolation from 31.8 percent in 1992 to 17 percent in 1996.14 This may be due to aggressive surveillance measures undertaken and an increased awareness among healthcare workers regarding good infection control practices. This study correlates well with our study.

In this Study Staphylococcus, Enterococcus, Streptococcus showed prevalence in this southern part of India, which varies from area to area. So individual Speciation does not seem to have much of a role in changing trends towards antibiotic policy.

\section{CONCLUSION}

In this 3-year study, Staphylococcus aureus was the most common organism isolated from clinical specimens. Staphylococcus aureus isolates showed high sensitivity to Glycopeptides Clindamycin, Doxycycline, Cefazolin, Erythromycin and Amoxicillin-Clavulanic acid. Enterococcus isolates showed high sensitivity to Glycopeptides. Methicillin Resistant Staphylococcus aureus and Vancomycin Resistant Enterococci exhibit lesser resistance rates in this 3-year study period. This study will help both clinicians and infection controlling practitioners to treat the patients. This single center study can give important information regarding emerging resistance patterns.

\section{Limitations}

This is a single centered study.

\section{ACKNOWLEDGEMENT}

The Authors thank the Dean and Director of Institute of Microbiology, Madurai Medical College, Madurai, who were kind enough to allow us to conduct this study.

The authors gratefully acknowledge the technicians and other members who have helped me to conduct this study successfully.

\section{REFERENCES}

1. Jeljaszewicz J, Mlynarczyk G, Mlynarczyk A. Antibiotic resistance in gram-positive cocci. Journal of AntiMicrobial Agents 2000;16(4):473-8.

2. Tong SY, Davis JS, Eichenberger E, et al. Staphylococcus aureus infections: epidemiology, pathophysiology, clinical manifestations, and management. Clinical Microbiology Reviews 2015;28(3):603-61.

3. Patel AK, Patel KK, Patel KR, et al. Time trends in the epidemiology of microbial infections at a tertiary care center in west India over last 5 years. J Assoc Physicians India 2010;58(Suppl):37-40. 
4. Gopalakrishnan R, Sureshkumar D. Changing trends in antimicrobial susceptibility and hospital acquired infections over an 8 year period in a tertiary care hospital in relation to introduction of an infection control programme. J Assoc Physicians India 2010;58(Suppl): 25-31.

5. Assadullah S, Kakru DK, Thoker MA, et al. Emergence of low level vancomycin resistance in MRSA. Indian J Med Microbiol 2003;21(3):196-8.

6. Centers for Disease Control and Prevention. Active Bacterial Core Surveillance Report. Emerging Infections Program Network. Streptococcus pneumoniae, 2013.

7. CLSI Performance standards for antimicrobial susceptibility testing. CLSI approved standard M100-S23. Clinical and Laboratory Standards Institute, Wayne, PA 19087 USA. 2013.

8. Kirby WM, Yoshihara GM, Sundsted KS, et al. Clinical usefulness of a single disc method for antibiotic sensitivity testing. Antibiotics Annu 1956-1957:892-7.

9. Indian Network for Surveillance of Antimicrobial Resistance (INSAR) group, India. Methicillin resistant staphylococcus aureus (MRSA) in India: prevalence \& susceptibility pattern. Indian J Med Res 2013;137(2): 363-9.
10. Goswami NN, Trivedi HR, Goswami APP, et al. Antibiotic sensitivity profile of bacterial pathogens in postoperative wound infections at a tertiary care hospital in Gujarat, India. Journal of Pharmocology and Pharamacotherpeutics 2011;2(3):158-64.

11. Mathur P, Kapil A, Chandra A, et al. Antimicrobial resistance in enterococcus fecalis at tertiary care center of Northern India. IJMR 2003;118:25-8.

12. Sreeja S, Sreenivasa Babu PR, Prathab AG. The prevalence and the characterization of the enterococcus species from various clinical samples in a tertiary care hospital. Journal of Clinical and Diagnostic Research 2012;6(9):1486-8.

13. Ratheesh R, Mohandas B, Sahadevan $P$, et al. A retrospective analysis on antimicrobial sensitivity pattern in a medical college hospital in Kannur district. Journal of Evolution of Medical and Dental Sciences 2015;4(37): 6443-50.

14. Mehta AP, Rodrigues C, Seth K, et al. Control of methicillin resistant staphylococcus aureus in a tertiary care centre: a five year study. IJMM 1998;16(1):31-4. 